Supporting Information

\title{
Synthesis of Organic Photodimeric Cage Molecules Based on Cycloaddition via Metal- Ligand Directed Assembly
}

Jarrod F. Eubank ${ }^{\dagger}{ }^{\dagger}$ Victor Ch. Kravtsov, ${ }^{\dagger, \uparrow}$ and Mohamed Eddaoudi ${ }^{*}, \uparrow$

Department of Chemistry, University of South Florida, 4202 E Fowler Ave (CHE205), Tampa, Florida 33620

Institute of Applied Physics of Academy of Sciences of Moldova, Academy str. 5, MD2028 Chisinau, Moldova

\section{Materials and Methods.}

Single-crystal X-ray diffraction data were collected on a Bruker SMART-APEX CCD diffractometer using $\mathrm{Mo}_{\mathrm{K} \alpha}$ radiation $(\lambda=0.71073 \AA)$.

XRPD data were recorded on a Rigaku RU15 diffractometer at $30 \mathrm{kV}, 15 \mathrm{~mA}$ for $\mathrm{Cu}_{\mathrm{K} \alpha}(\lambda=1.5418 \AA)$, with a scan speed of $1 \% \mathrm{~min}$ and a step size of $0.05^{\circ}$ in $2 \theta$ at room temperature.

Photodimerization reactions were carried out in a Rayonet RPR-600 Photochemical Reactor.

Preparation for 1: 3,5- $\mathrm{H}_{2} \mathrm{PDC}(13.4 \mathrm{mg}, 0.08 \mathrm{mmol}), \mathrm{Co}\left(\mathrm{NO}_{3}\right)_{2} \cdot 6 \mathrm{H}_{2} \mathrm{O}(46.6 \mathrm{mg}$, $0.16 \mathrm{mmol})$, EtOH $(1 \mathrm{~mL}), \operatorname{DMF}(1 \mathrm{~mL})$, Py $(0.05 \mathrm{~mL})$ were added to a $20 \mathrm{~mL}$ scintillation vial, and the solution was heated at a constant rate $1.5^{\circ} \mathrm{C} / \mathrm{min}$ to $85^{\circ} \mathrm{C}$ for 12 $\mathrm{h}$, then cooled at a constant rate $1{ }^{\circ} \mathrm{C} / \mathrm{min}$ to room temperature. Fuchsia block crystals were collected and air-dried, and were insoluble in $\mathrm{H}_{2} \mathrm{O}$ and common organic solvents.

Preparation for 2: $\mathrm{H}_{2} \mathrm{CDO} \cdot \mathrm{H}_{2} \mathrm{O}(8.1 \mathrm{mg}, 0.04 \mathrm{mmol}), \mathrm{Cu}\left(\mathrm{NO}_{3}\right)_{2} \cdot 2.5 \mathrm{H}_{2} \mathrm{O}(9.3 \mathrm{mg}$, $0.04 \mathrm{mmol}), \mathrm{DMF}(1 \mathrm{~mL})$, EtOH $(1 \mathrm{~mL})$, Py $(0.1 \mathrm{~mL})$ were added to a $20 \mathrm{~mL}$ scintillation vial, and the solution was heated at a constant rate $1.5^{\circ} \mathrm{C} / \mathrm{min}$ to $85^{\circ} \mathrm{C}$ for 12 $\mathrm{h}$, then cooled at a constant rate $1{ }^{\circ} \mathrm{C} / \mathrm{min}$ to room temperature. Light blue plate crystals 
were collected and air-dried (15 mg, 90\% yield), and were insoluble in $\mathrm{H}_{2} \mathrm{O}$ and common organic solvents.

Preparation for 3: Method 1: Crystals of 2 in a $20 \mathrm{~mL}$ scintillation vial were washed with DMF $(1 \mathrm{~mL})$ and $\mathrm{EtOH}(1 \mathrm{~mL})$ three times, and the final solution with crystals placed in a photochemical reactor for 1 day (until crystals turn from light blue to light green). Light green plate crystals were collected and air-dried, and were insoluble in $\mathrm{H}_{2} \mathrm{O}$ and common organic solvents.

Method 2: Crystals of 2 in a $20 \mathrm{~mL}$ scintillation vial were washed with DMF (1 $\mathrm{mL})$ and $\mathrm{EtOH}(1 \mathrm{~mL})$ three times and dried. The crystals were distributed in a thin layer on a recrystallization dish and placed in a photochemical reactor for 1 day (until crystals turn from light blue to light green). Light green plate crystals were collected, and were insoluble in $\mathrm{H}_{2} \mathrm{O}$ and common organic solvents.

Preparation for 4: Crystals of 3 in a $20 \mathrm{~mL}$ scintillation vial were washed with DMF $(1 \mathrm{~mL})$ and EtOH $(1 \mathrm{~mL})$ three times and dried. The crystals were then washed with $\mathrm{H}_{2} \mathrm{O}$ three times and dried, followed by the addition of $\mathrm{HNO}_{3}(1.0 \mathrm{~mL} 6 \mathrm{~N}$ in $1.0 \mathrm{~mL}$ $\mathrm{H}_{2} \mathrm{O}$ ). The solution was heated at a constant rate $1.5^{\circ} \mathrm{C} / \mathrm{min}$ to $85^{\circ} \mathrm{C}$ for $12 \mathrm{~h}$, then cooled at a constant rate $1{ }^{\circ} \mathrm{C} / \mathrm{min}$ to room temperature, followed by successive heating at a constant rate $1.5^{\circ} \mathrm{C} / \mathrm{min}$ to $105^{\circ} \mathrm{C}$ for $23 \mathrm{~h}$ and cooling at a constant rate $1^{\circ} \mathrm{C} / \mathrm{min}$ to room temperature. Blue rod crystals were collected from the side wall of the vial.

Preparation for 5: Crystals of 3 in a $20 \mathrm{~mL}$ scintillation vial were washed with DMF $(1 \mathrm{~mL})$ and EtOH $(1 \mathrm{~mL})$ three times and dried. The crystals were then washed with $\mathrm{H}_{2} \mathrm{O}$ three times and dried, followed by the addition of $\mathrm{NaOH}\left(1.0 \mathrm{~mL} 8 \mathrm{M}\right.$ in $\left.\mathrm{H}_{2} \mathrm{O}\right)$. The blue/green precipitate was filtered and concentrated $\mathrm{HNO}_{3}$ added to $\sim \mathrm{pH} 2.0$, and 
placed at $4^{\circ} \mathrm{C}$ for three days. Colorless plate crystals were collected from the vial and air dried ( $8 \mathrm{mg}, 34 \%$ total yield based on $\mathrm{H}_{2} \mathrm{CDO} \cdot \mathrm{H}_{2} \mathrm{O}$ ).

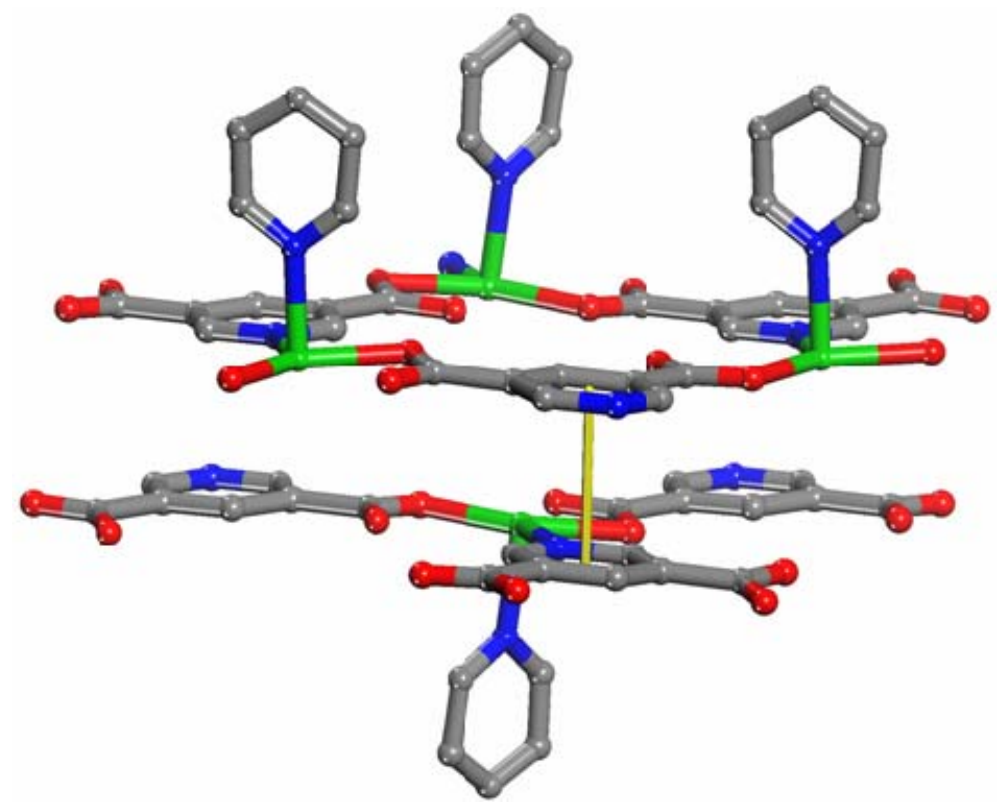

Figure S1. Analysis of the single-crystal structure of our Co-(3,5-PDC) MOF, 1, shows alternating $2 \mathrm{D}$ honeycomb or $(6,3)$ layers. The organic linkers are positioned in an anti conformation along the $\mathrm{x}$ axis with centroid...centroid distances of $7.47 \AA$ and $3.55 \AA$, respectively. The latter distance (yellow) is within the appropriate range, in addition to the proper positioning of organic linkers, for [2+2] photodimerization. Water molecules and hydrogen atoms have been omitted for clarity; cobalt = green, carbon = gray; nitrogen $=$ blue; oxygen $=$ red. 
a)
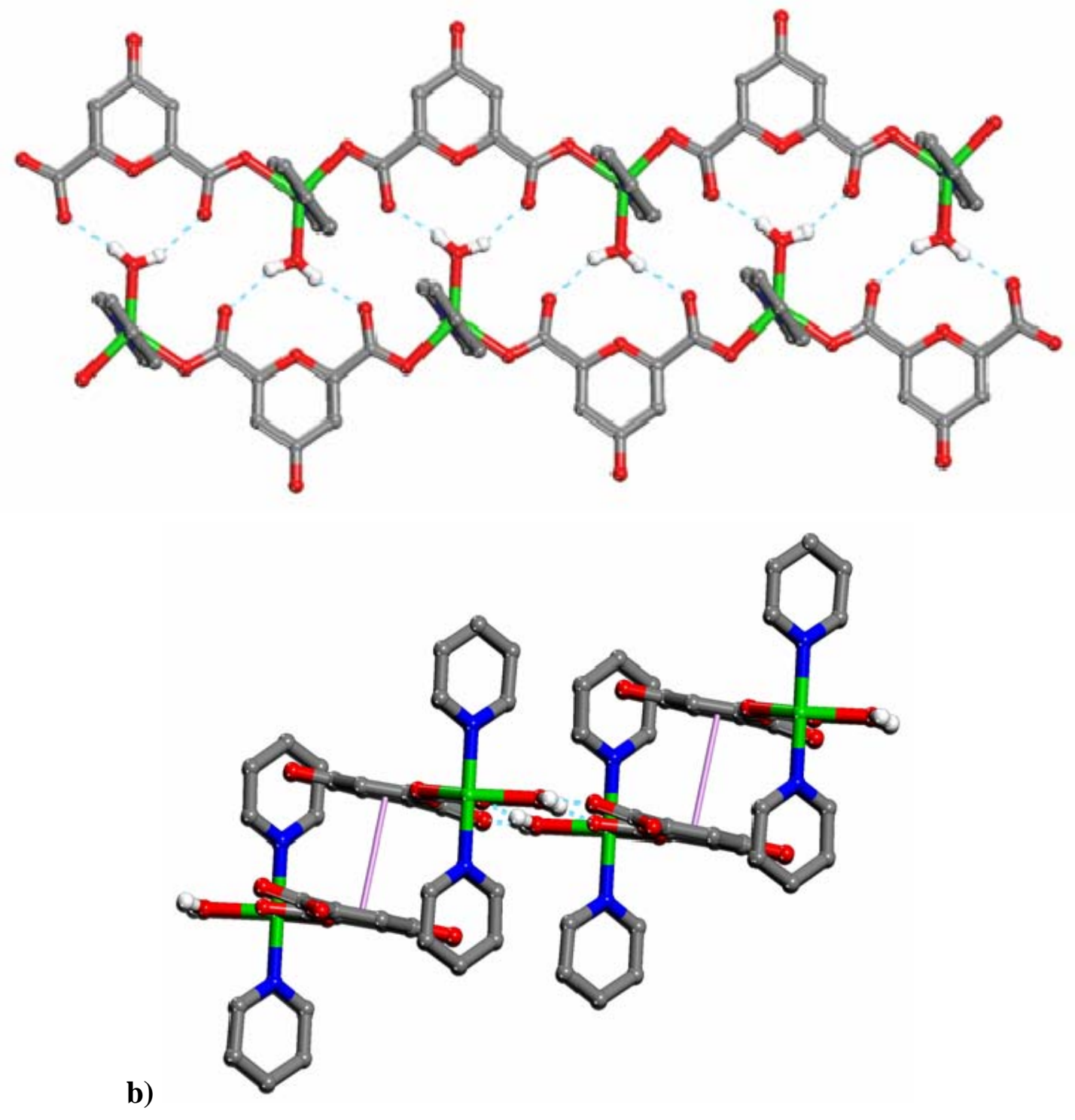

Figure S2. Single-crystal structure of 2: a) the metal-organic chains of $\mathbf{2}$ hydrogen bond to neighboring chains through the coordinated water molecules, resulting in metalorganic ladders, and b) are separated by axial terminal pyridine molecules and held in close proximity by CDO...CDO interactions (centroid...centroid distance represented in fuschia). Excepting water molecules, hydrogen atoms have been omitted for clarity; copper $=$ green, carbon $=$ gray; nitrogen $=$ blue; oxygen $=$ red. 


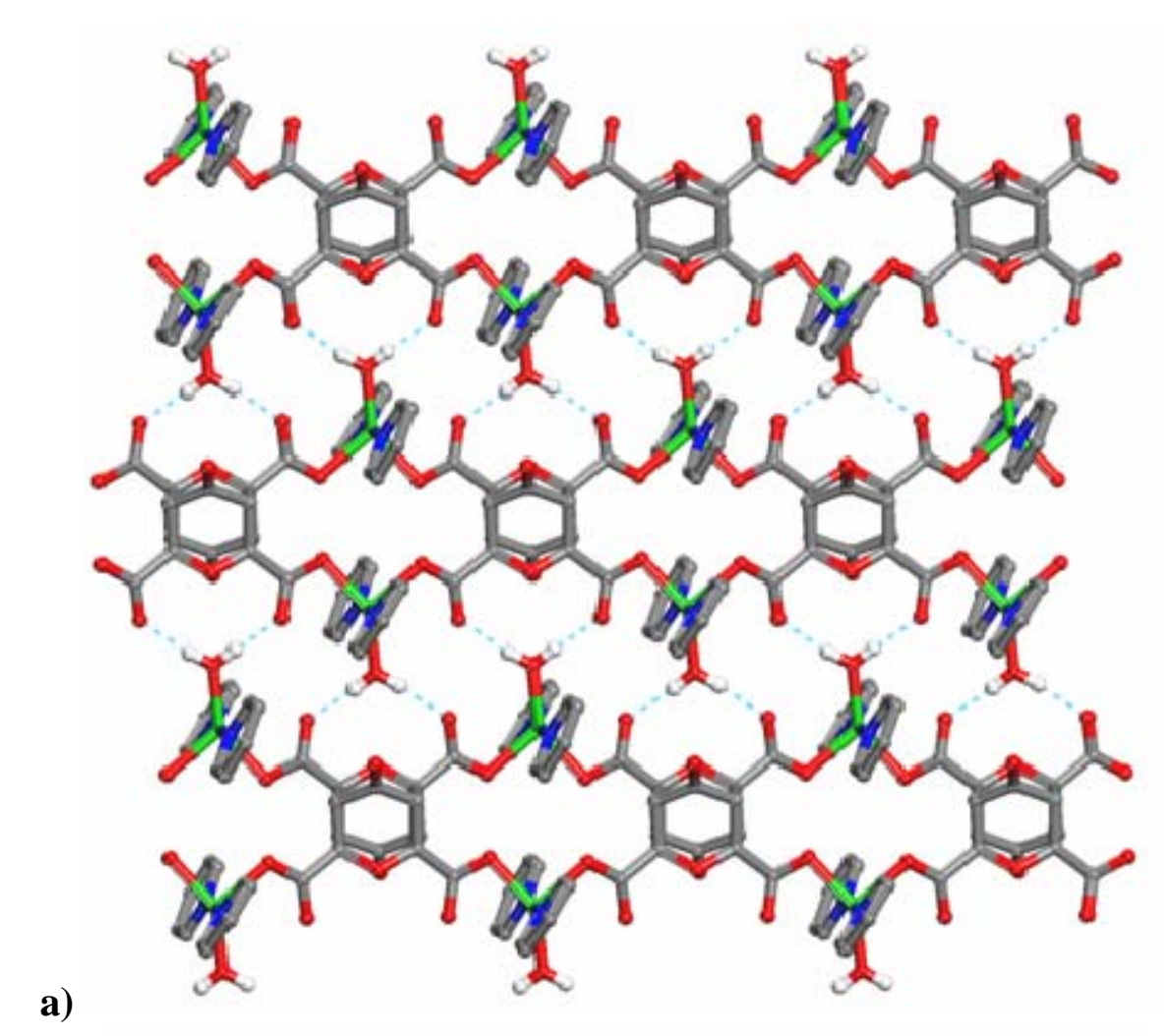

a)

b)

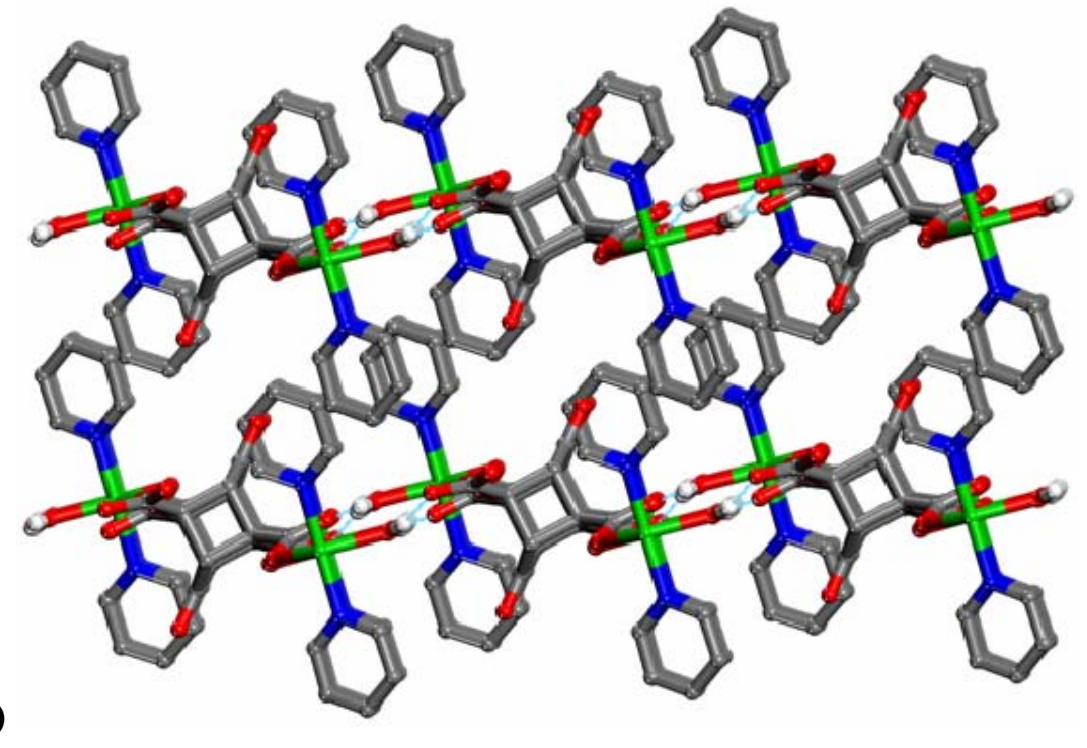

Figure S3. Single-crystal structure of 3: a) [2+2] photodimerized CDO ligands, EE1 (Eubank-Eddaoudi-1), in 3 result in metal-organic ladders that hydrogen bond to neighboring ladders to form 2D layers, and b) these layers are separated by the axial terminal pyridine molecules. Excepting water molecules, hydrogen atoms have been omitted for clarity; copper $=$ green, carbon $=$ gray; nitrogen $=$ blue; oxygen $=$ red. 

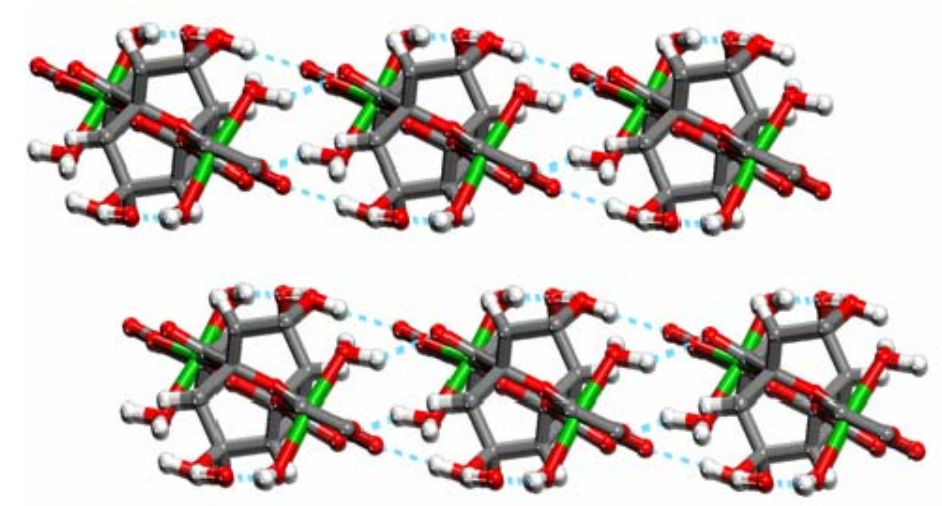

a)

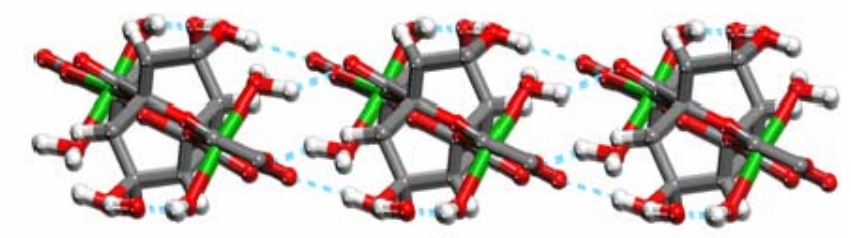

b)

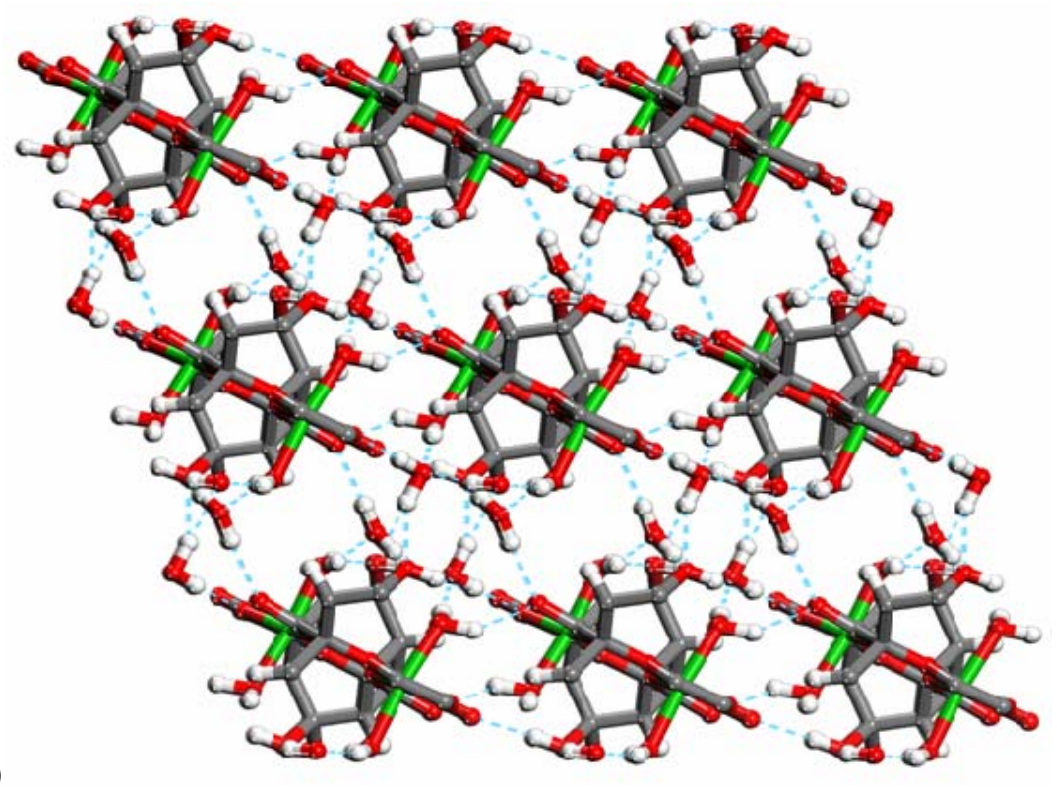

Figure S4. Single-crystal structure of 4: a) the metal-organic ladders of 4 are hydrogen bonded through coordinated water molecules to form 2D layers, and b) these layers are hydrogen bonded to guest water molecules to form a 3D structure. Copper $=$ green, carbon $=$ gray; oxygen $=$ red. 

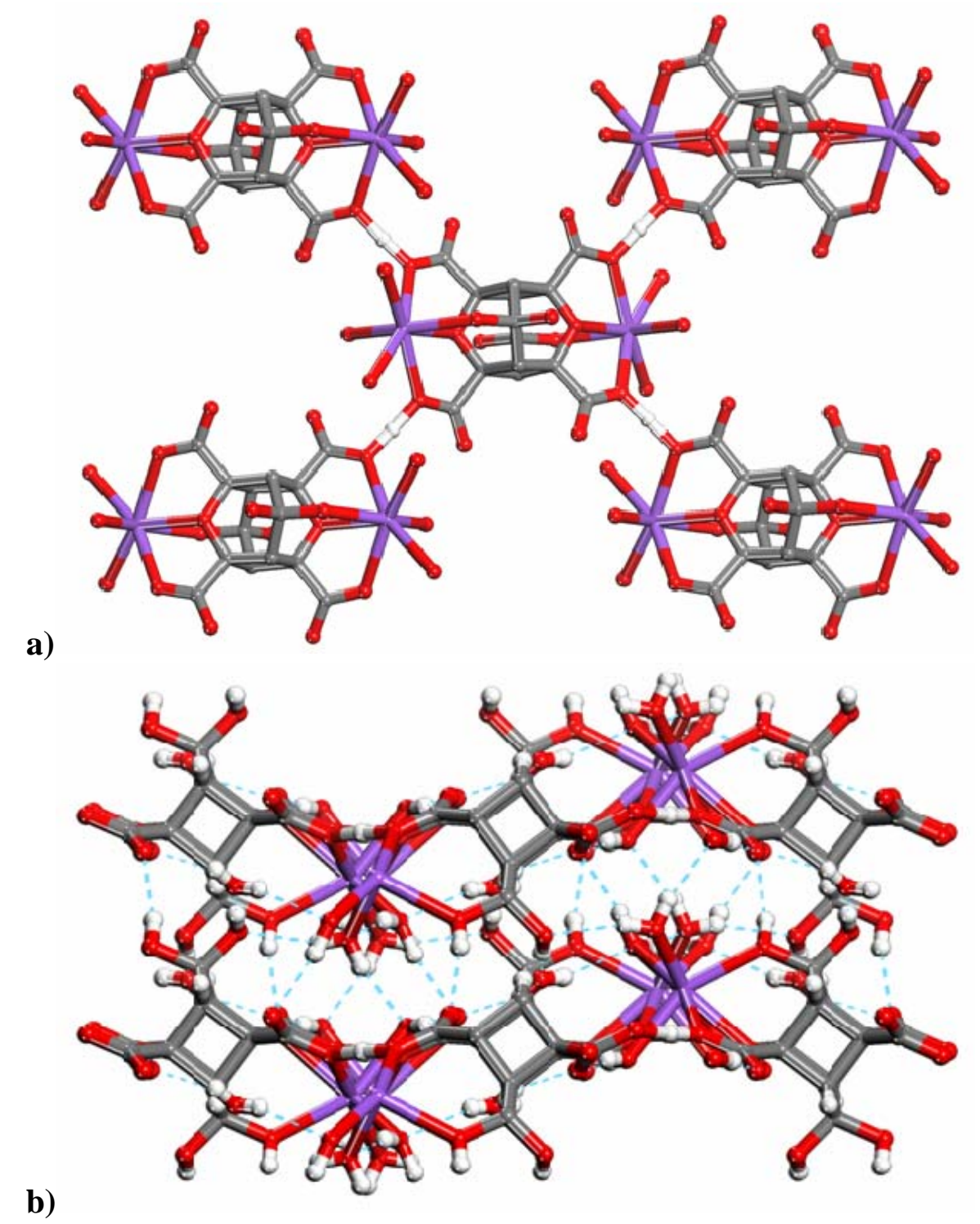

Figure S5. Single-crystal structure of 5: a) multiple O-H...O HBs between ligands in 5 unite the individual EE2 (Eubank-Eddaoudi-2) molecules into layers having 4.4 square grid topology, indicating the potential use of EE2 as a 4-connected linker in MOFs, and b) the additional O-H...O HBs between ligands and water molecules result in a $3 \mathrm{D}$ organic framework. Sodium $=$ violet, carbon $=$ gray; oxygen $=$ red. 


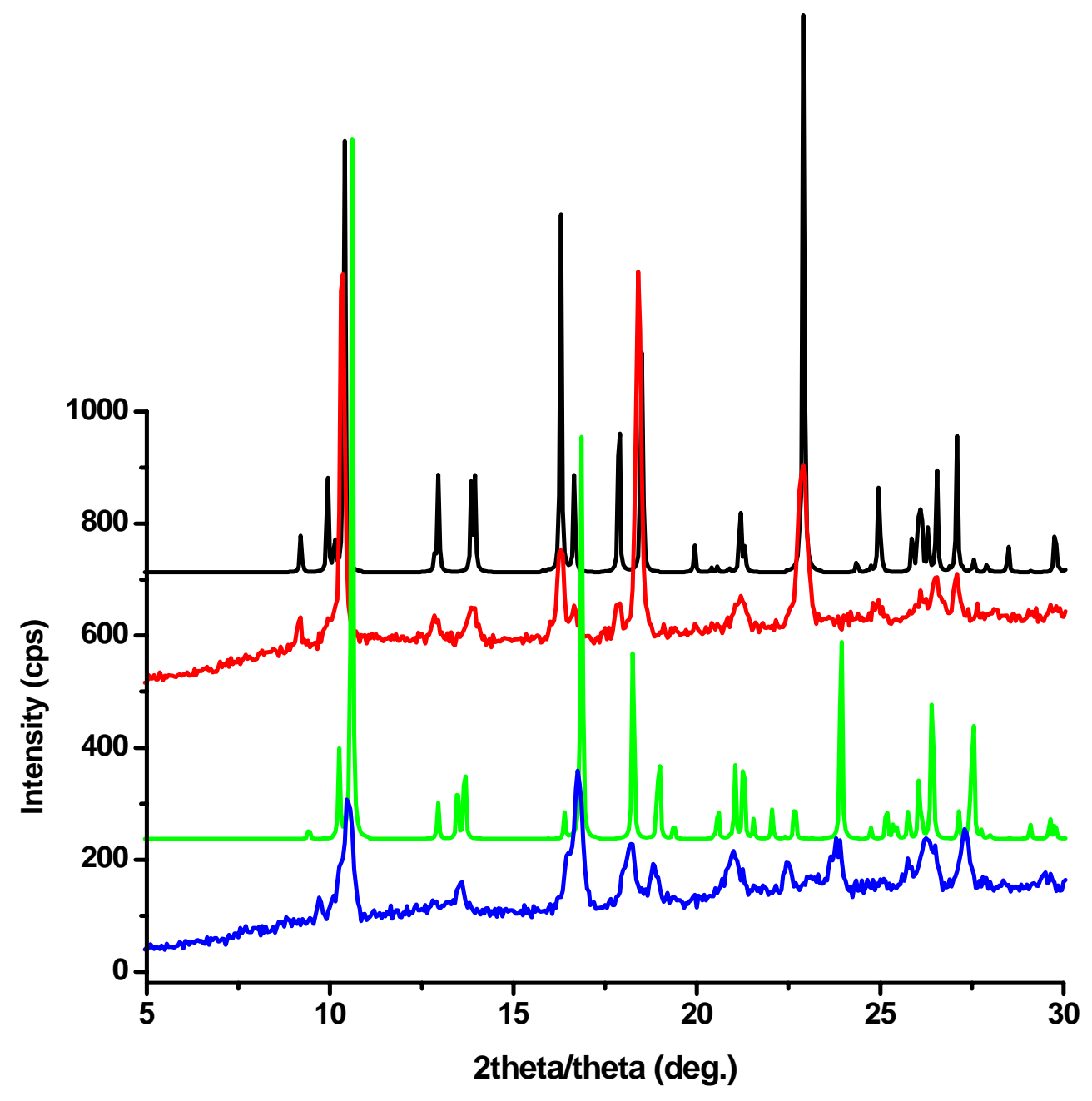

Figure S6. XRPD patterns for 2 (black = simulated, red = experimental), and 3 (green = simulated, blue $=$ experimental). 
Table S1. Crystal data and structure refinement for 1, 2, and 3.

\begin{tabular}{|c|c|c|c|}
\hline Compound & 1 & 2 & 3 \\
\hline Empirical formula & $\mathrm{CoC}_{12} \mathrm{H}_{10} \mathrm{~N}_{2} \mathrm{O}_{5}$ & $\mathrm{CuC}_{17} \mathrm{H}_{14} \mathrm{~N}_{2} \mathrm{O}_{7}$ & $\mathrm{CuC}_{17} \mathrm{H}_{4} \mathrm{~N}_{2} \mathrm{O}_{7}$ \\
\hline Formula weight & 321.15 & 421.84 & 411.76 \\
\hline Temperature & $100(2) \mathrm{K}$ & $100(2) \mathrm{K}$ & $293(2) \mathrm{K}$ \\
\hline Wavelength & $0.71073 \AA$ & $0.71073 \AA$ & $0.71073 \AA$ \\
\hline Crystal system & Monoclinic & Triclinic & Triclinic \\
\hline Space group & $P 2(1) / \mathrm{c}$ & $P-1$ & $P-1$ \\
\hline Unit cell dimensions & $\begin{array}{c}\mathrm{a}=10.8654(14) \AA \\
\mathrm{b}=9.8741(12) \AA \\
\mathrm{c}=11.9102(14) \AA \\
\alpha=90^{\circ} \\
\beta=91.465(2)^{\circ} \\
\gamma=90^{\circ}\end{array}$ & $\begin{array}{c}\mathrm{a}=8.738(3) \AA \\
\mathrm{b}=10.188(4) \AA \\
\mathrm{c}=10.760(4) \AA \\
\alpha=63.080(7)^{\circ} \\
\beta=83.713(8)^{\circ} \\
\gamma=76.770(7)^{\circ}\end{array}$ & $\begin{array}{c}\mathrm{a}=8.586(2) \AA \\
\mathrm{b}=9.954(3) \AA \\
\mathrm{c}=10.576(3) \AA \\
\alpha=62.738(4)^{\circ} \\
\beta=87.681(4)^{\circ} \\
\gamma=77.070(4)^{\circ}\end{array}$ \\
\hline Volume & $1277.4(3) \AA^{3}$ & $831.5(5) \AA^{3}$ & $781.1(3) \AA^{3}$ \\
\hline $\mathrm{Z}$ & 4 & 2 & 2 \\
\hline Density (calculated) & $1.670 \mathrm{mg} / \mathrm{m}^{3}$ & $1.685 \mathrm{mg} / \mathrm{m}^{3}$ & $1.751 \mathrm{mg} / \mathrm{m}^{3}$ \\
\hline Abs. coefficient & $1.364 \mathrm{~mm}^{-1}$ & $1.359 \mathrm{~mm}^{-1}$ & $1.445 \mathrm{~mm}^{-1}$ \\
\hline $\mathrm{F}(000)$ & 652 & 430 & 410 \\
\hline$\theta$ range for data collect. & 1.87 to $25.08^{\circ}$ & 2.29 to $26.50^{\circ}$ & 2.17 to $26.99^{\circ}$ \\
\hline Index ranges & $\begin{array}{l}-12 \leq \mathrm{h} \leq 10 \\
-11 \leq \mathrm{k} \leq 10 \\
-14 \leq \mathrm{l} \leq 13\end{array}$ & $\begin{array}{l}-10 \leq \mathrm{h} \leq 10 \\
-12 \leq \mathrm{k} \leq 12 \\
-12 \leq \mathrm{l} \leq 13\end{array}$ & $\begin{array}{c}-10 \leq \mathrm{h} \leq 10 \\
-12 \leq \mathrm{k} \leq 12 \\
-13 \leq \mathrm{l} \leq 8\end{array}$ \\
\hline Reflections collect. & 6606 & 4890 & 4661 \\
\hline Independent reflect. & $2258[\mathrm{R}(\mathrm{int})=0.0434]$ & $3359[\mathrm{R}(\mathrm{int})=0.0536]$ & $3231[\mathrm{R}(\mathrm{int})=0.1838]$ \\
\hline Complete. to $\theta \max$ & $99.6 \%$ & $97.3 \%$ & $94.8 \%$ \\
\hline Abs. correction & SADABS & None & None \\
\hline Refinement method & $\begin{array}{l}\text { Full-matrix least-sq. } \\
\text { on } \mathrm{F}^{2}\end{array}$ & $\begin{array}{l}\text { Full-matrix least-sq. } \\
\text { on } \mathrm{F}^{2}\end{array}$ & $\begin{array}{c}\text { Full-matrix least-sq. } \\
\text { on } \mathrm{F}^{2}\end{array}$ \\
\hline Data/restraints/param. & $2258 / 0 / 218$ & $3359 / 2 / 250$ & $3231 / 22 / 250$ \\
\hline Goodness-of-fit on $\mathrm{F}^{2}$ & 1.065 & 0.939 & 1.002 \\
\hline $\begin{array}{l}\text { Final } R \text { indices } \\
{[\mathrm{I}>2 \operatorname{sigma}(\mathrm{I})]}\end{array}$ & $\begin{array}{c}\mathrm{R} 1=0.0418 \\
\mathrm{wR} 2=0.1048\end{array}$ & $\begin{array}{c}\mathrm{R} 1=0.0597 \\
\mathrm{wR} 2=0.0913\end{array}$ & $\begin{array}{c}\mathrm{R} 1=0.0543 \\
\mathrm{wR} 2=0.0954\end{array}$ \\
\hline $\mathrm{R}$ indices (all data) & $\begin{array}{c}\mathrm{R} 1=0.0529 \\
\mathrm{wR} 2=0.1104\end{array}$ & $\begin{array}{c}\mathrm{R} 1=0.0999 \\
\mathrm{wR} 2=0.1033\end{array}$ & $\begin{array}{c}\mathrm{R} 1=0.1449 \\
\mathrm{wR} 2=0.1383\end{array}$ \\
\hline Largest diff. peak/hole & $0.673 /-0.375$ e. $\mathrm{A}^{-3}$ & $0.605 /-0.646$ e. $\mathrm{A}^{-3}$ & $1.475 /-1.507 \mathrm{e} . \mathrm{A}^{-3}$ \\
\hline
\end{tabular}


Table S2. Crystal data and structure refinement for $\mathbf{4}$ and $\mathbf{5 .}$

\begin{tabular}{|c|c|c|}
\hline Compound & 4 & 5 \\
\hline Empirical formula & $\mathrm{CuC}_{7} \mathrm{H}_{12} \mathrm{~N}_{2} \mathrm{O}_{11}$ & $\mathrm{Na}_{2} \mathrm{C}_{14} \mathrm{H}_{26} \mathrm{O}_{22}$ \\
\hline Formula weight & 363.73 & 592.33 \\
\hline Temperature & $100(2) \mathrm{K}$ & $100(2) \mathrm{K}$ \\
\hline Wavelength & $0.71073 \AA$ & $0.71073 \AA$ \\
\hline Crystal system & Triclinic & Monoclinic \\
\hline Space group & $P-1$ & $P 21 / \mathrm{c}$ \\
\hline Unit cell dimensions & $\begin{array}{l}a=7.729(3) \AA \\
b=8.805(3) \AA \\
c=9.393(3) \AA \\
\alpha=62.493(6)^{\circ} \\
\beta=81.809(6)^{\circ} \\
\gamma=68.261(6)^{\circ}\end{array}$ & $\begin{array}{c}\mathrm{a}=7.297(3) \AA \\
\mathrm{b}=16.347(7) \AA \\
\mathrm{c}=11.990(4) \AA \\
\alpha=90^{\circ} \\
\beta=126.824(17)^{\circ} \\
\gamma=90^{\circ}\end{array}$ \\
\hline Volume & $526.3(3) \AA^{3}$ & $1144.9(8) \AA^{3}$ \\
\hline $\mathrm{Z}$ & 2 & 2 \\
\hline Density (calculated) & $2.295 \mathrm{mg} / \mathrm{m}^{3}$ & $1.718 \mathrm{mg} / \mathrm{m}^{3}$ \\
\hline Abs. coefficient & $2.153 \mathrm{~mm}^{-1}$ & $0.196 \mathrm{~mm}^{-1}$ \\
\hline $\mathrm{F}(000)$ & 370 & 616 \\
\hline$\theta$ range for data collect. & 2.77 to $26.49^{\circ}$ & 2.46 to $25.49^{\circ}$ \\
\hline Index ranges & $\begin{array}{c}-9 \leq \mathrm{h} \leq 9 \\
-10 \leq \mathrm{k} \leq 11 \\
-11 \leq \mathrm{l} \leq 7\end{array}$ & $\begin{array}{c}-8 \leq \mathrm{h} \leq 8 \\
-19 \leq \mathrm{k} \leq 19 \\
-14 \leq \mathrm{l} \leq 12\end{array}$ \\
\hline Reflections collect. & 2947 & 5963 \\
\hline Independent reflect. & $2094[\mathrm{R}(\mathrm{int})=0.0945]$ & $2106[\mathrm{R}(\mathrm{int})=0.1597]$ \\
\hline Complete. to $\theta \max$ & $96.1 \%$ & $99.0 \%$ \\
\hline Abs. correction & None & None \\
\hline Refinement method & $\begin{array}{c}\text { Full-matrix least-sq. } \\
\text { on } \mathrm{F}^{2}\end{array}$ & $\begin{array}{c}\text { Full-matrix least-sq. } \\
\text { on } \mathrm{F}^{2}\end{array}$ \\
\hline Data/restraints/param. & $2094 / 14 / 177$ & $2106 / 0 / 203$ \\
\hline Goodness-of-fit on $\mathrm{F}^{2}$ & 1.000 & 1.009 \\
\hline $\begin{array}{l}\text { Final } R \text { indices } \\
{[\mathrm{I}>2 \operatorname{sigma}(\mathrm{I})]}\end{array}$ & $\begin{array}{c}\mathrm{R} 1=0.0524 \\
\mathrm{wR} 2=0.1248\end{array}$ & $\begin{array}{c}\mathrm{R} 1=0.0613 \\
\mathrm{wR} 2=0.1615\end{array}$ \\
\hline $\mathrm{R}$ indices (all data) & $\begin{array}{c}\mathrm{R} 1=0.0861 \\
\mathrm{wR} 2=0.1454\end{array}$ & $\begin{array}{c}\mathrm{R} 1=0.0653 \\
\mathrm{wR} 2=0.1645\end{array}$ \\
\hline Largest diff. peak/hole & $1.414 /-1.358 \mathrm{e} . \mathrm{A}^{-3}$ & $0.580 /-0.536 \mathrm{e} \cdot \mathrm{A}^{-3}$ \\
\hline
\end{tabular}

